\title{
Three Dimensional Optimum Node Localization in Dynamic Wireless Sensor Networks
}

\section{Gagandeep Singh Walia ${ }^{1}$, Parulpreet Singh ${ }^{1}$, Manwinder Singh ${ }^{1}$, Mohamed Abouhawwash ${ }^{2,3}$, Hyung Ju Park ${ }^{4}$, Byeong-Gwon Kang, ,", Shubham Mahajan ${ }^{5}$ and Amit Kant Pandit ${ }^{5}$}

\author{
${ }^{1}$ Department of Electronics and Communication Engineering, Lovely Professional University, Jalandhar, 144411, \\ Punjab, India \\ ${ }^{2}$ Department of Mathematics, Faculty of Science, Mansoura University, Mansoura, 35516, Egypt \\ ${ }^{3}$ Department of Computational Mathematics, Science, and Engineering (CMSE), Michigan State University, East Lansing, \\ MI, 48824, USA \\ ${ }^{4}$ Department of ICT Convergence, Soonchunhyang University, Asan, 31538, Korea \\ ${ }^{5}$ School of Electronics \& Communication, Shri Mata Vaishno Devi University, Katra, 182320, India \\ ${ }^{*}$ Corresponding Author: Byeong-Gwon Kang. Email: bgkang@sch.ac.kr \\ Received: 03 April 2021; Accepted: 04 May 2021
}

\begin{abstract}
Location information plays an important role in most of the applications in Wireless Sensor Network (WSN). Recently, many localization techniques have been proposed, while most of these deals with two Dimensional applications. Whereas, in Three Dimensional applications the task is complex and there are large variations in the altitude levels. In these 3D environments, the sensors are placed in mountains for tracking and deployed in air for monitoring pollution level. For such applications, 2D localization models are not reliable. Due to this, the design of 3D localization systems in WSNs faces new challenges. In this paper, in order to find unknown nodes in Three-Dimensional environment, only single anchor node is used. In the simulation-based environment, the nodes with unknown locations are moving at middle \& lower layers whereas the top layer is equipped with single anchor node. A novel soft computing technique namely Adaptive Plant Propagation Algorithm (APPA) is introduced to obtain the optimized locations of these mobile nodes. These mobile target nodes are heterogeneous and deployed in an anisotropic environment having an Irregularity (Degree of Irregularity (DOI)) value set to 0.01 . The simulation results present that proposed APPA algorithm outperforms as tested among other meta-heuristic optimization techniques in terms of localization error, computational time, and the located sensor nodes.
\end{abstract}

Keywords: Wireless sensor networks; localization; particle swarm optimization; h-best particle swarm optimization; biogeography-based optimization; grey wolf optimizer; firefly algorithm; adaptive plant propagation algorithm 


\section{Introduction}

Wireless Sensor Networks (WSNs) contain many small low-power sensor nodes (SNs) deployed randomly in the environment to determine the physical behavior. Sensors are often used to obtain measurements of location, temperature, humidity, irradiance, sound, and pressure [1]. In most of WSNs applications location determination is crucially important and sensor nodes deployed in these areas are of utmost importance as no one is present in the field to locate and place the nodes personally. So, in these applications sensor nodes are randomly deployed at unknown locations and they adopt random locations in the sensor field. On the other hand, the exact location is not known of an occurring event the information gathered by these sensors is useless [2]. To locate the sensor nodes in WSN, GPS which is one of the most widely used technique for localization, was developed to overcome the limitations of previous navigation systems [2]. GPS is being used in military, industry, and more recently, consumer/civilian applications. However, GPS does not work with obstacles that limit LOS communications between the satellites and the GPS receiver, therefore, its utility is limited in dense forests, mountains, and also in indoor environments. To overcome GPS limitations, sensor networks can be applied for localization. An alternative way to find out all unknown nodes in the scenario is to deploy few sensors within built GPS feature in them are known as anchor nodes. Thus, the exact location of these sensors is known after deployment in WSNs. By using the known locations of these anchors, many methods already available in literature are used for evaluation of the location of unknown nodes (or unknown nodes). Range-based and Range-free algorithms are different algorithms that exist in the literature. First one measures the distance between nodes using RSSI, AoA, ToA [3,4]. Thus, range free strategies, distance vector hop, multidimensional signaling, and adhoc positioning system provide the location of various targeted nodes with fewer infrastructure requirements. In WSN, providing exact localization is one of the greatest problems. Localization can be done precisely in static nodes, but it is much more difficult in moving nodes. We introduced the idea of using a novel APPA to target unknown nodes with the help of only one node which is called as the anchor and assumption is taken about this node virtually in six different directions. Whenever the nodes whose location is to be found outcomes under the range of anchor, virtual anchors placed at 60 degrees' angles, with the same range as that of anchor, and out of the six only three nodes are nominated to trace the exact position of the unknown node because at least four SNs are needed to find out three dimensional positions. Here, we are working to find out the evaluation and hence efficiency of localization problem with various meta-heuristics using APPA.

The following section in this work is as described: Section 2 illustrates challenges which deal with 3D localization. Section 3, introduces a novel approach named APPA. In Section 4 the process of deploying only one anchor node in the sensing field is explained. Section 5 concludes results and discussions. At last, the Future Scope and the conclusive part is discussed in Section 6.

\section{Literature Review}

A lot of research is done in Wireless Communications. Liu et al. [5,6] have published several papers on a variety of wireless networks, including Mobile Ad Hoc Social Networks and Mobile Opportunistic Networks. This paper, in comparison to their network, focuses on WSNs with multiple sensor nodes to track a physical area. Various localization schemes have recently been proposed, with the majority of research proposals concentrating on Two Dimensional localization techniques with a flat sensing region. Due to this, the design of Three Dimensional localization systems in WSNs faces new challenges. 
Chu et al. [7] developed a new global optimization algorithm called the Symbiotic Organism Search Algorithm with Multi-Group Quantum-Behavior Communication (MQSOS) by integrating the multi-group communication and quantum behavior strategies with the symbiotic organisms search (SOS) algorithm. It is swift and convergent, and it is useful for solving practical problems involving multiple arguments. Under the CEC2013 large-scale optimization test suite, they compared MQSOS to other intelligent algorithms including particle swarm optimization (PSO), parallel PSO (PPSO), adaptive PSO (APSO), Quasi-Affine Transformation Evolutionary (QUATRE), and oppositional SOS (OSOS). The results of the experiments show that the MQSOS algorithm outperformed the other intelligent algorithms. Liu et al. [8] proposed various strategies to accomplish the localization of nodes using distance data between neighbor nodes. they verify through experiment that the proposed algorithms provide better performance in confinement accuracy and energy utilization. Distributed localization nodes, according to Kotwal et al. [9], use crude RSSI to estimate their minimum and maximum distance limits with respect to anchor nodes. A simple binary search algorithm is used in the approximation. The rough distance limits assist in the creation of the node's feasibility area in relation to anchor nodes. To solve the optimization problem of minimizing localization error, the feasibility area coordinates are used as initial particles in particle swarm optimization (PSO). It was discovered that nodes can be localized with greater accuracy using simple calculations than current algorithms, and that fewer anchor nodes with limited communication range are needed. In a wireless sensor network (WSN) system, Low et al. [10] present a localization system for unknown emitter nodes. For this scheme, four anchor nodes with known positions are presumed, as well as one or more unknown nodes transmitting RF signals that can be received by the four anchor nodes. The system's only source of data is the obtained signal strength indicator, which is inaccurate. The particle swarm optimization (PSO) scheme, which can be implemented in real time, is investigated in this paper to obtain a better approximate position of the sensor nodes. The suggested approach's simulation and experimental findings are discussed. To improve the WSN localization accuracy, Wang et al. [11] proposed a new coupling algorithm based on Bacterial Foraging Algorithm (BFA) and Glowworm Swarm Optimisation (GSO) (BFO-GSO). The algorithm has good convergence speed and the optimization performance is verified by CEC2013 benchmarks. The RSSI method is used to measure the estimated distance between the reference and target nodes deployed in the field using the trilateration approach, according to Graefenstein et al. [12]. Sumathi et al. [13] proposed an RSS method for locating unknown nodes that only needs a single anchor node. This paper presents a least squares method for locating fixed target nodes. Guo et al. [14] developed a mobilebased method called perpendicular intersection (PI) that does not map RSS distances directly. The geometric PI relationship is used to calculate the location of the node. Shi et al. [15] proposed a scheme, in which a single mobile anchor sends ultra wideband (UWB) signals to the sensor nodes for localizing whole network The Distance Vector-Hop dependent approach for locating sensor nodes was introduced by Wang et al. [16]. The failure of this algorithm is primarily due to its complexity and increased cost. Xu et al. [17] proposed an improved 3D localization technique that combined DV-Distance with the quasi-newton optimize approach to improve the performance. The efficacy of the proposed algorithm was further checked by taking into account localization accuracy and coverage. The 3D WSN localization approach based on irregular RSSI model was proposed by Li et al. [18]. The authors proposed this model to quantify the relationship between DOIs and signal transmission range variability. When the deployed sensors are positioned in an area surrounded by a community of anchor nodes, Ahmad et al. [19] proposed a parametric loop-division algorithm for 3D localization. This approach accurately shrinks the network toward the center and produces reliable localization performance. Gopakumar et al. [20] proposed a new 
and computationally efficient swarm intelligence method for locating static nodes that is easy to implement and requires little memory. Chuang et al. [21] use the RSS ranging technique to efficiently locate sensor nodes using a PSO-based approach. In terms of localization, the scheme has a higher success rate. PSO-Iterative is a distributed iterative localization algorithm developed by Kulkarni et al. [22,23]. There are more than three anchors for each target node, and PSO is used to reduce the localization error. Kumar et al. [24] proposed localization strategies based on HPSO and BBO principles with minimal hardware specifications, dubbed Range free HPSO and $\mathrm{BBO}$, respectively. The edge weights are optimized using PSO and BBO applications. In order to optimize the position of unknown sensor nodes, Arora et al. [25] suggested using the BOA optimization algorithm. The performance of PSO and FA in 2D scenarios is compared to the performance of BOA. As compared to other meta heuristic algorithms, their solution outperforms in terms of convergence time and position accuracy. Range-based methods are widely used due to their higher precision, but flip uncertainty is a major disadvantage to range-based methods. References [26-30] proposed a PSO-based computational intelligence algorithm for determining the position of moving target nodes in WSNs. The algorithm is divided into two stages, with anchor nodes placed at the corners of the sensing area. During the first step, distance calculations were made using RSSI. Virtual anchor nodes were believed to locate unknown nodes with the aid of anchor in a later stage. In these stages, centroid calculations are obtained along with an optimization technique called PSO, and the results indicate a faster convergence time. The APPA algorithm has been used to deal with the localization principle in WSNs in this article. The main goal is to investigate the efficiency of the APPA algorithm in the localization of WSNs and compare it to the output of other algorithms. The following segment explains the basic concept behind APPA.

\section{Adaptive Plant Propagation Algorithm (APPA)}

This Algorithm is comprised of a population of shoots, and every shoot presents a solution in the search space. It is assumed that each shoot has taken root which is equivalent to the objective function being assessed. Each shoot will then send runners out to explore the space around the solution.

A plant is considered to be in a location $Y_{i}=\left\{y_{i, j}, j=1,2, \ldots, n\right\}$, where the dimension of the search space is given as $n$. Let the population size be denoted as $\mathrm{N}_{\mathrm{p}}$ which determines the number of strawberry plants to be used initially. It is known that Strawberry plants which are in poor spots propagate by sending long runners which are few in number, the process being known as exploration. The plants which are in location with abundance of essential nutrients, minerals and water propagate by sending many short runners, the process is known as exploitation. Maximum number of generations considered is $\mathrm{g}_{\max }$ and maximum number of permissible runners per plant is $\mathrm{n}_{\max }$.

The objective function values at different positions $\mathrm{Y}_{\mathrm{i}}, \mathrm{i}=1,2, \ldots, \mathrm{N}_{\mathrm{p}}$ are calculated. These possible candidate solutions will be sorted according to their fitness scores. Here the fitness is a function of value of the objective function under consideration. It is better to keep the fitness scores within ascertain boundary between 0 and 1 , that is, $f(x) \in[0,1]$. To keep the fitness values within this range, a mapping is done using the sigmoid function, described by Eq. (1)

$$
\mathrm{N}(\mathrm{x})=\frac{\exp \left(\frac{(\mathrm{f}(\mathrm{x}))}{\max (\mathrm{f}(\mathrm{x}))}\right)}{1+\left(\exp \left(\frac{(\mathrm{f}(\mathrm{x}))}{\max (\mathrm{f}(\mathrm{x}))}\right)\right)}
$$


The effect of this mapping function is that, it provides a means of emphasizing further better solutions over those which are not as good.

The number of runners that are found out by the solution and the distance of propagation of each of them are described. There exists a direct relationship between the number of runners produced by a candidate solution and its fitness given by Eq. (2)

$\mathrm{n}_{\mathrm{r}}=\operatorname{ceil}\left(\mathrm{n}_{\max } \mathrm{N}_{\mathrm{i}} \mathrm{r}\right)$

Here, $\mathrm{n}_{\mathrm{r}}$ is the number of runners produced for solution $\mathrm{i}$ in a particular generation or iteration after the population is sorted according to the fitness given in Eq. (2), $\mathrm{n}_{\max }$ is the number of runners which is maximum permissible, $\mathrm{N}_{\mathrm{i}}$ is the mapped fitness as determined using Eq. (2), $\mathrm{r}$ is a random number lying between 0 and 1 which is randomly selected for each individual in every iteration or generation, and ceil refers to the ceiling function. The minimum number of runners is 1 and maximum is $n_{r}$. This function ensures that at least 1 runner should be there which may correspond to the long runner as described before. The distance of each runner is inversely related to its fitness as shown in Eq. (3)

$\mathrm{d}_{\mathrm{j}}^{\mathrm{i}}=\left(1-\mathrm{N}_{\mathrm{i}}\right)(\mathrm{r}-0.5)$ for $\mathrm{j}=1,2, \ldots, \mathrm{n}$

where $\mathrm{n}$ represents the dimension of the search space. So, each runner is restricted to a certain range between -0.5 and 0.5 . The calculated distance of the runners is used to update the solution for further exploration and exploitation of the search space by the Eq. (4)

$x_{i, j}=y_{i, j}+\left(b_{j}-a_{j}\right) d_{j}^{i} \quad$ for $j=1,2, \ldots, n$

The algorithm is modified to be an adaptive one in view of the limits of the search domain. Hence, the name is given as Adaptive Plant Propagation Algorithm (APPA). In the event that the limits are disregarded the point is changed in accordance to lie within the search space. Essentially, $a_{j}$ and $b_{j}$ are the lower and upper boundaries of the $j$ th coordinate of the search space respectively. New plants are polled and the entire extended population is organized after every single individual plant in the population has passed on their designated runners. To keep the population fixed, rather than the size of the population fixed, it is to be guaranteed that the candidates with lower growth are dispensed from the population. Another strategy is adopted to avoid being struck in the local minima. It might happen that for a certain number of generations there is no improvement in a candidate solution, rather the runners it sends out are also not fit to remain in the population. So a threshold to be set for such a solution such that if the number of generations in which it is not enhancing surpasses the threshold then the solution is discarded and another fresh candidate solution or individual is produced within the limits of the search space.

\section{Single Anchor Node Localization Concept}

In this 3D localization problem, a single anchor node with known location information is considered and this location information of anchor is utilized to find out the locations of randomly placed mobile nodes. These mobile nodes are grouped into three different layers with anchor placed at the top most position and unknown nodes are moving at middle and the bottom layers. Anchor nodes transmits a beacon signal that will be sensed by mobile nodes and using the concept of virtual anchors, three of these virtual anchors and anchor node itself are selected to locate all the mobile nodes. Based on received RSSI the approximated distance between anchor 
and target node is estimated. The complete flow of localization procedure is given by Fig. 1. The detailed description of localization using APPA algorithm is given in Fig. 2.

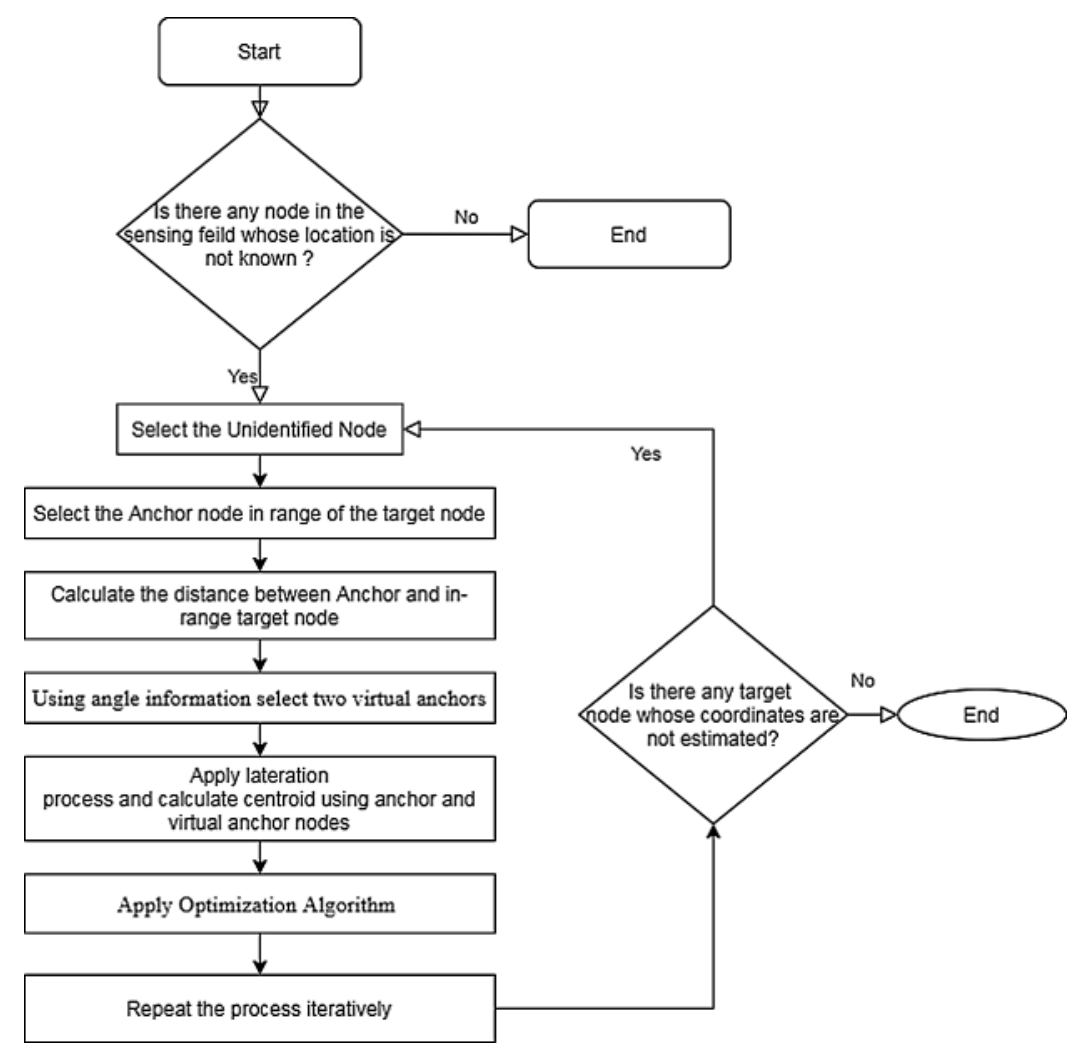

Figure 1: Flowchart of optimized localization using Adaptive Plant Propagation Algorithm (APPA)

The proposed algorithm has below mentioned properties and further steps for estimating location information have been discussed in this section.

a) Using the APPA algorithm, a new method for projecting virtual nodes in the field to determine the exact locations of deployed sensor nodes in a three dimensional scenario.

b) Line of Sight (LoS) problems will be reduced to a greater extent with virtual anchor nodes.

c) Flip ambiguity issues in range-based methods are also minimized.

Firstly, the anchor and moving targets distance is determined in $3 \mathrm{D}$ scenarios using RSS measures. Further, the anchor nodes which are virtual (six in number) are placed with same distance at an angle difference of sixty degrees, given by Fig. 3. For each target localization, anchor with three virtual anchor nodes is selected in order to find coordinates in three dimensional scenarios respectively, shown by Fig. 4. This selection of virtual anchor nodes is done using directional information of target node. The anchor and target node distance is given by Eq. (5)

$\mathrm{d}_{\mathrm{i}}=\sqrt{\left(\mathrm{x}_{\mathrm{t}}-\mathrm{x}\right)^{2}+\left(\mathrm{y}_{\mathrm{t}}-\mathrm{y}\right)^{2}+\left(\mathrm{z}_{\mathrm{t}}-\mathrm{z}\right)^{2}}$ 

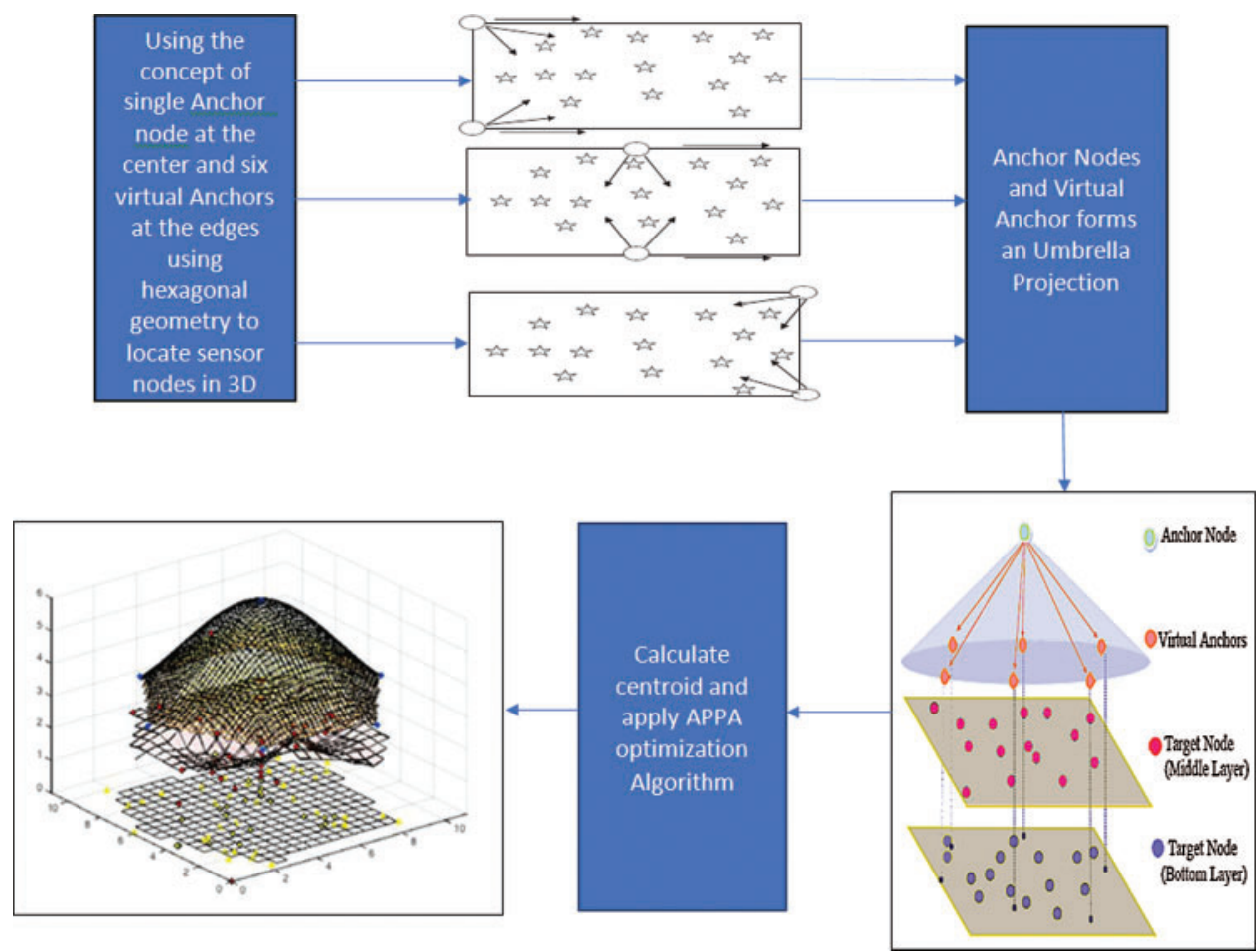

Figure 2: Detailed description of 3D localization using Adaptive Plant Propagation Algorithm (APPA)

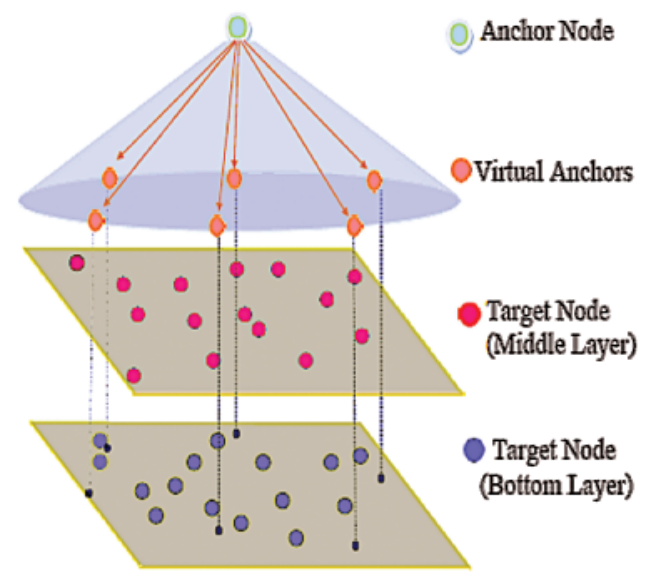

Figure 3: Umbrella projection to find out the position of mobile target nodes

Here in three dimensions, the position of the nodes which are targets is given by $\left(x_{t}, y_{t}, z_{t}\right)$ and the present location of the anchor node is given by $(x, y, z)$ for three dimensional scenarios. Further, the centroid $\left(x_{c}, y_{c}, z_{c}\right)$ is obtained by Eq. (6) in three dimensional environments, and is given in Fig. 5. Each moving target localize itself using APPA with the centroid value (xc, yc, zc) as the initial guess 
$\left(\mathrm{x}_{\mathrm{c}}, \mathrm{y}_{\mathrm{c}}, \mathrm{z}_{\mathrm{c}}\right)=\left(\frac{\mathrm{x}+\mathrm{xv}_{1}+\mathrm{xv}_{2}+x \mathrm{v}_{3}}{3}, \frac{\mathrm{y}+\mathrm{yv}_{1}+\mathrm{yv}_{2}+y \mathrm{v}_{3}}{3}, \frac{\mathrm{z}+\mathrm{zv}_{1}+\mathrm{zv}_{2}+z \mathrm{v}_{3}}{3}\right)$

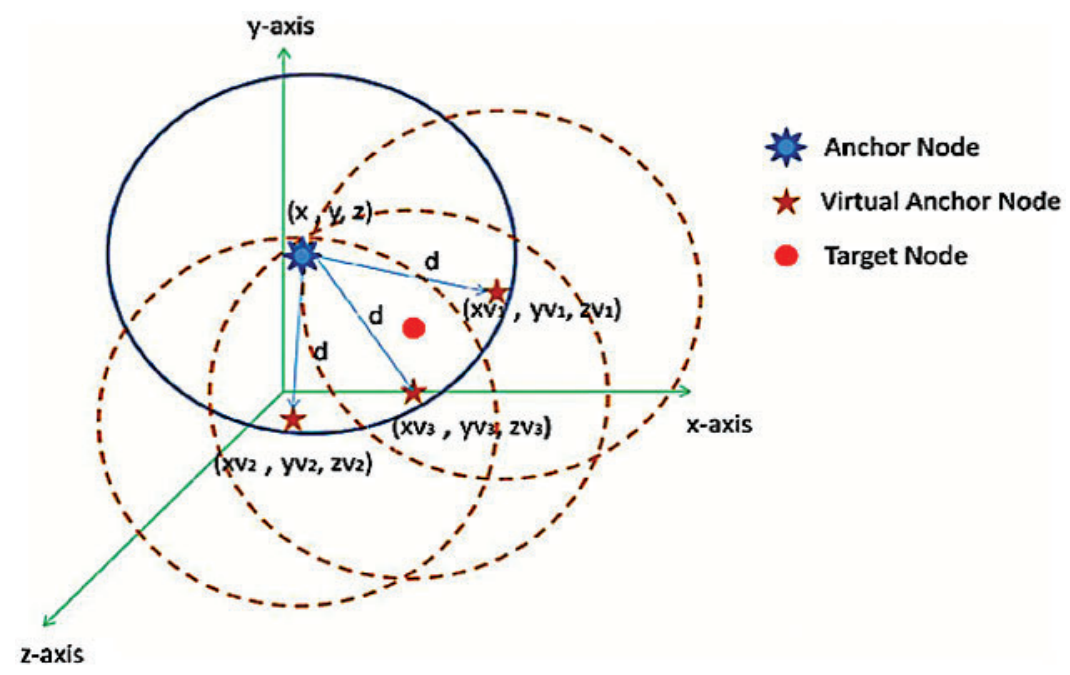

Figure 4: Sensor field in 3D environment with anchor and virtual anchor nodes

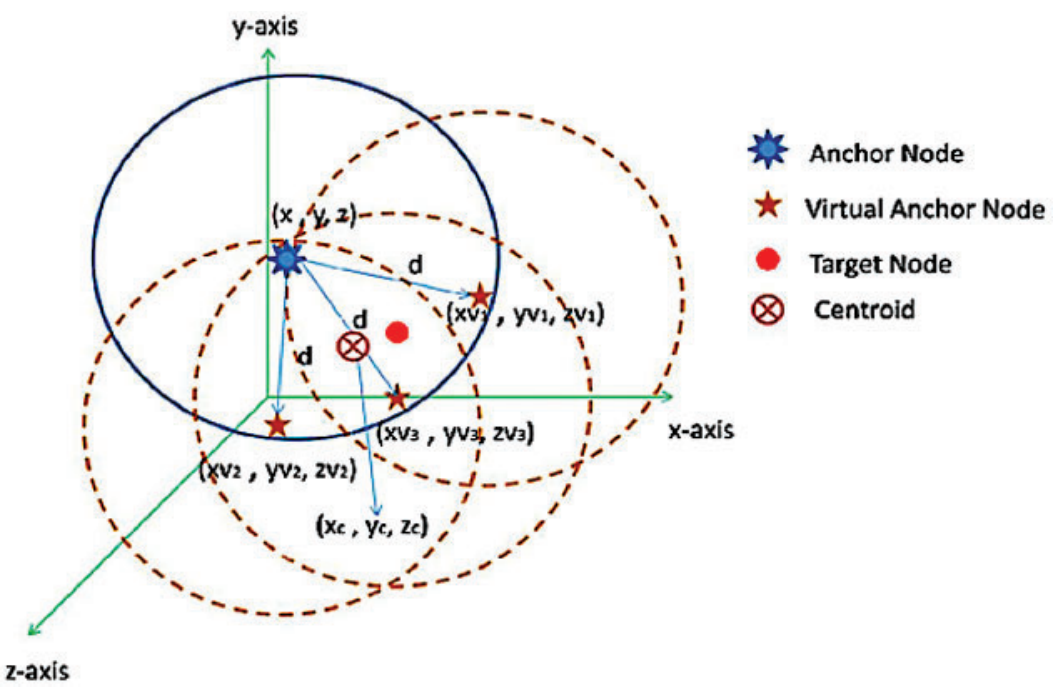

Figure 5: 3D centroid calculation

It has been shown by Fig. 6 that proposed APPA is used to find out the coordinates of the node which is the target and is given by $\left(\mathrm{x}_{\mathrm{s}}, \mathrm{y}_{\mathrm{s}}, \mathrm{z}_{\mathrm{s}}\right.$ ). The distance among the estimated and actual 
deployment of target nodes will be reduced by the objective function utilized in Eq. (7)

$\mathrm{f}\left(\mathrm{x}_{\mathrm{s}}, \mathrm{y}_{\mathrm{s}}, \mathrm{z}_{\mathrm{s}}\right)=\frac{1}{\mathrm{M}} \sum\left(\sqrt{\left(\mathrm{x}_{\mathrm{e}}-\mathrm{x}_{\mathrm{i}}\right)^{2}+\left(\mathrm{y}_{\mathrm{e}}-\mathrm{y}_{\mathrm{i}}\right)^{2}+\left(\mathrm{z}_{\mathrm{e}}-\mathrm{z}_{\mathrm{i}}\right)^{2}}-\mathrm{d}_{\mathrm{i}}^{\wedge}\right)^{2}$

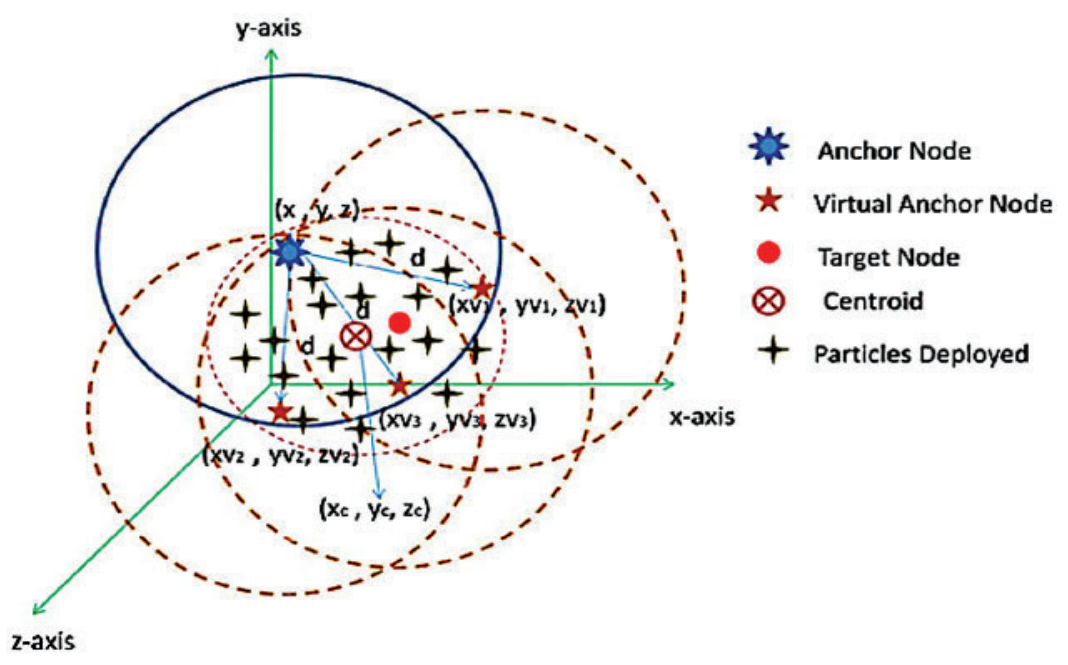

Figure 6: APPA particles deployed in 3D scenario

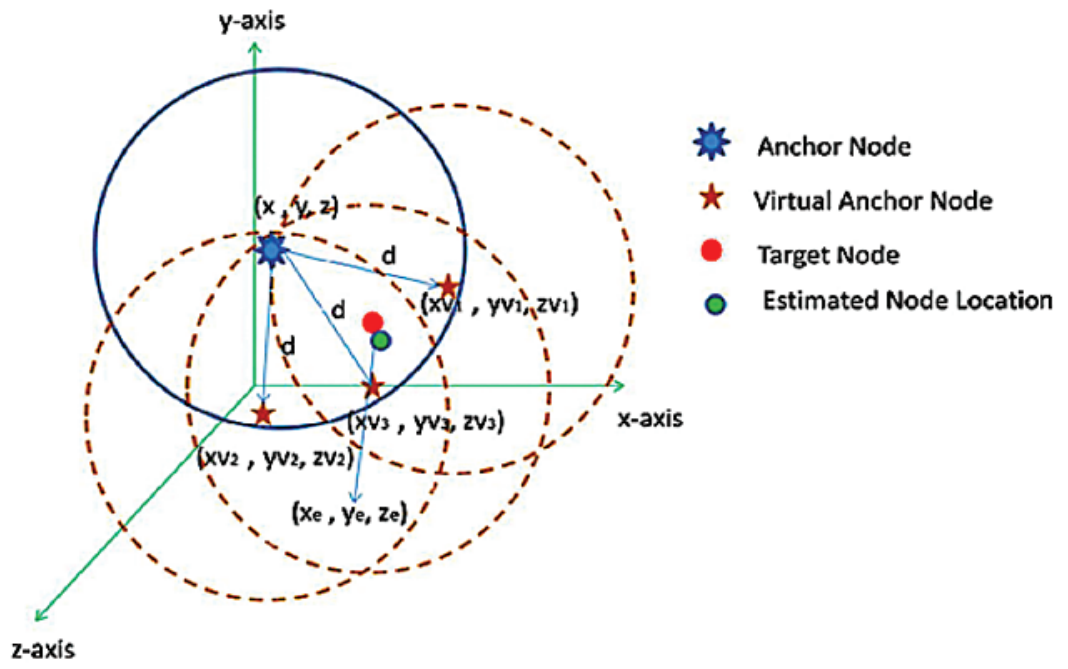

Figure 7: Estimated 3D location

Here, the estimated position of the target node is given by $\left(\mathrm{x}_{\mathrm{e}}, \mathrm{y}_{\mathrm{e}}, \mathrm{z}_{\mathrm{e}}\right)$, estimated position of the beacon node $i$ and the nodes which are the targets is given by $\left(x_{i}, y_{i}, z_{i}\right)(M>4$ to compute 3D location) respectively for $3 \mathrm{D}$ scenario. 
Error in the process of localization is given by $\mathrm{E}_{\mathrm{t}}$, and is found out by Eq. (8) and is shown in Fig. 7 for three dimensional scenarios.

$\mathrm{E}_{\mathrm{t}}=\frac{1}{\mathrm{~N}_{\mathrm{L}}} \sum \sqrt{\left(\mathrm{x}_{\mathrm{e}}-\mathrm{x}_{\mathrm{t}}\right)^{2}+\left(\mathrm{y}_{\mathrm{e}}-\mathrm{y}_{\mathrm{t}}\right)^{2}+\left(\mathrm{z}_{\mathrm{e}}-\mathrm{z}_{\mathrm{i}}\right)^{2}}$

\section{Simulation Results and Discussion}

Here, a novel technique APPA is used for three dimensional localization problem where the concept one anchor and six virtual anchors assumed in six directions placed at $60^{\circ}$ is considered to find out the exact position of all unknown nodes. In three dimensional environments, the structure is divided into different layers, normally three and it is a cubic structure. Here the unknown nodes whose position is to be found out are placed at the lower two layers and the known nodes are kept at the top most layer. The number of unknown nodes at each layer is kept to be forty. For finding out the positions of the unknown nodes in three dimensional environments, an umbrella projection is created. Deployment of more than six virtual anchors is also practically possible, but by keeping more than six virtual anchor nodes, hardly there is any change in the efficiency of the algorithm. The parameters required for various meta-heuristic optimization algorithms are given below in Tab. 1.

Table 1: Parameter settings

\begin{tabular}{ll}
\hline Algorithm & Parameters \\
\hline PSO & $N P=30 ; D=3 ; G_{\max }=100 ; c_{1}, c_{2}, c_{3}=1.494 ; w=0.729$ \\
HPSO & $N P=30 ; D=3 ; G_{\max }=100 ; c_{1}, c_{2}, c_{3}=1.494 ; \eta=0.1 ; w=0.729$ \\
BBO & $N P=30 ; D=3 ; G_{\max }=100 ; p_{m}=0.05$ \\
FA & $N P=30 ; D=3 ; G_{\max }=100 ; \alpha=0.2 ; \gamma=0.96$ \\
GWO & $N P=20 ; D=3 ; G_{\max }=100 ; \mathrm{a}=[2$ to 0$] ; \mathrm{C}=[0$ to 2$]$ \\
APPA & $N P=30 ; D=3 ; G_{\max }=100 ; n_{\max }=3$ \\
\hline
\end{tabular}

Here, NP is number of population, D is dimension of problem, Gmax is number of iteration.

Where (c1), (c2) and (c3) are the cognitive, social and neighborhood learning parameters. Here $\mathrm{W}$ is the inertia weight and Pm is the probability of mutation. In FA $\mathrm{x}$ and $\gamma$ are randomizing and absorption coefficient. In mobility-based scenario, various optimization algorithms available in the literature are evaluated. Here the unknown nodes whose position is to be found out are placed at the lower two layers and the known nodes are kept at the top most layer. All the unknown nodes are moving while the anchor node is kept static. The average of the localization error given in Eq. (8) is used to find out the fitness function. Figs. 8-13 represent the output obtained by various optimized algorithms. The line of sight disadvantage is also reduced a lot with the help of assuming various nodes at different angles. It has been proved with the help of the results that using APPA, accurate locations are being found as compared to other algorithms and convergence characteristics are also faster. In future, with the help of hybridization of few optimized algorithms more accuracy could be achieved. 


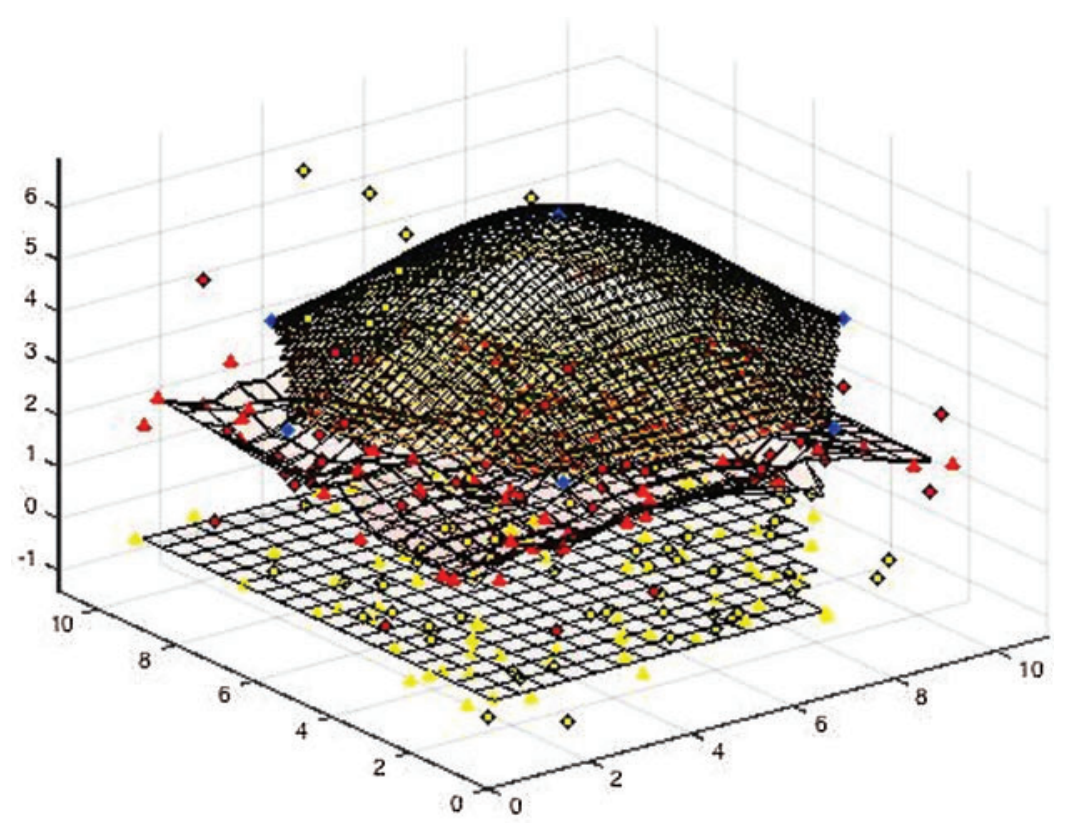

Figure 8: Optimized localization of sensor nodes with BBO

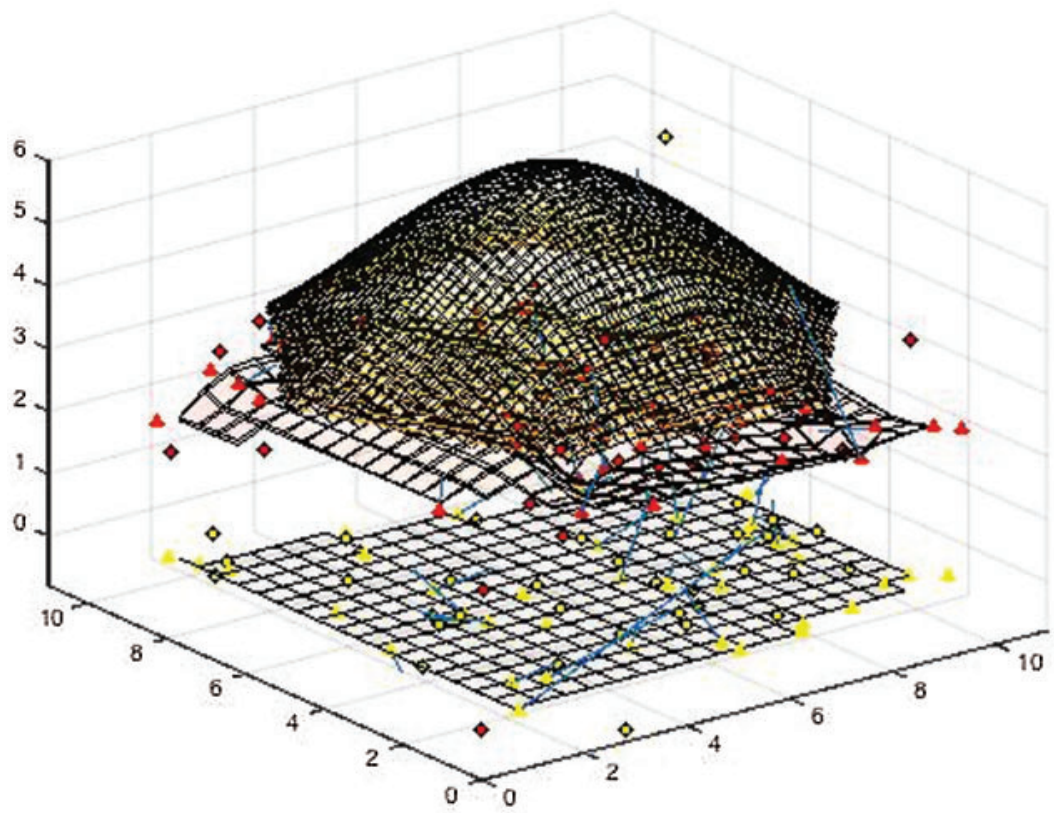

Figure 9: Optimized localization of sensor nodes with PSO

The average localization error for all competitive algorithms is computed in Tab. 2 and shown in Fig. 14. When compared to other competitive algorithms tested for the same situation, APPA has a much faster convergence time. 


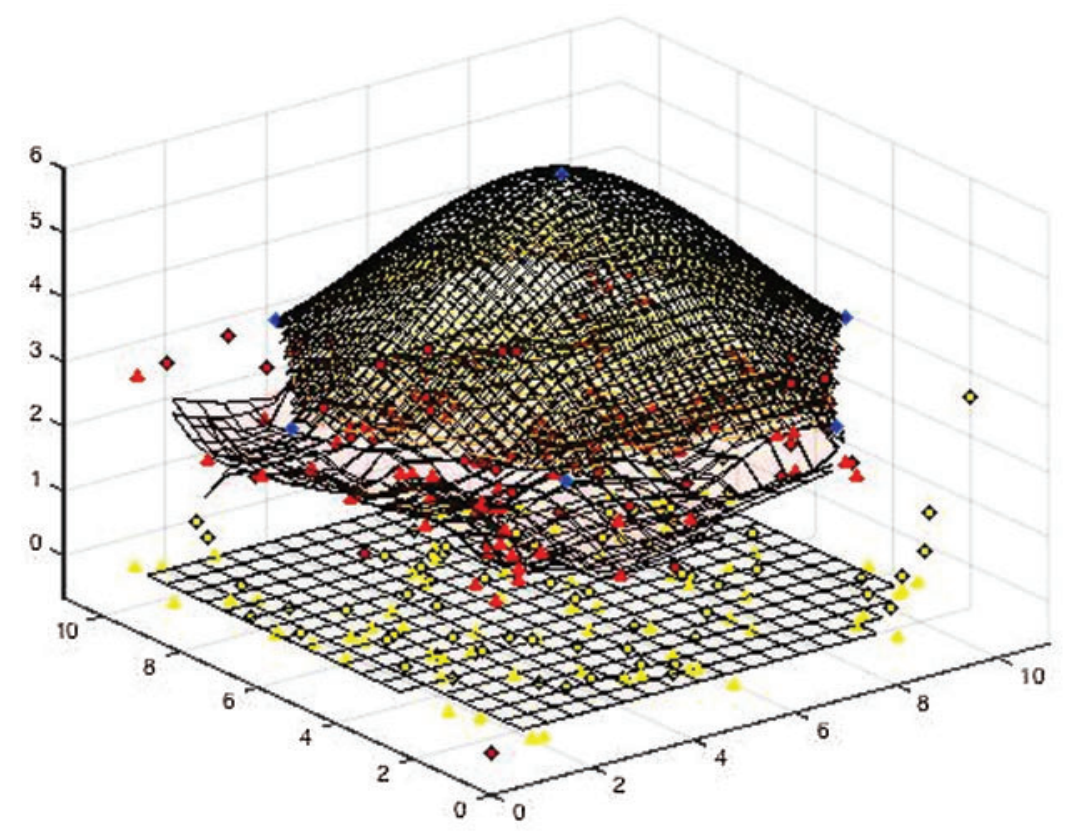

Figure 10: Optimized localization of sensor nodes with FA

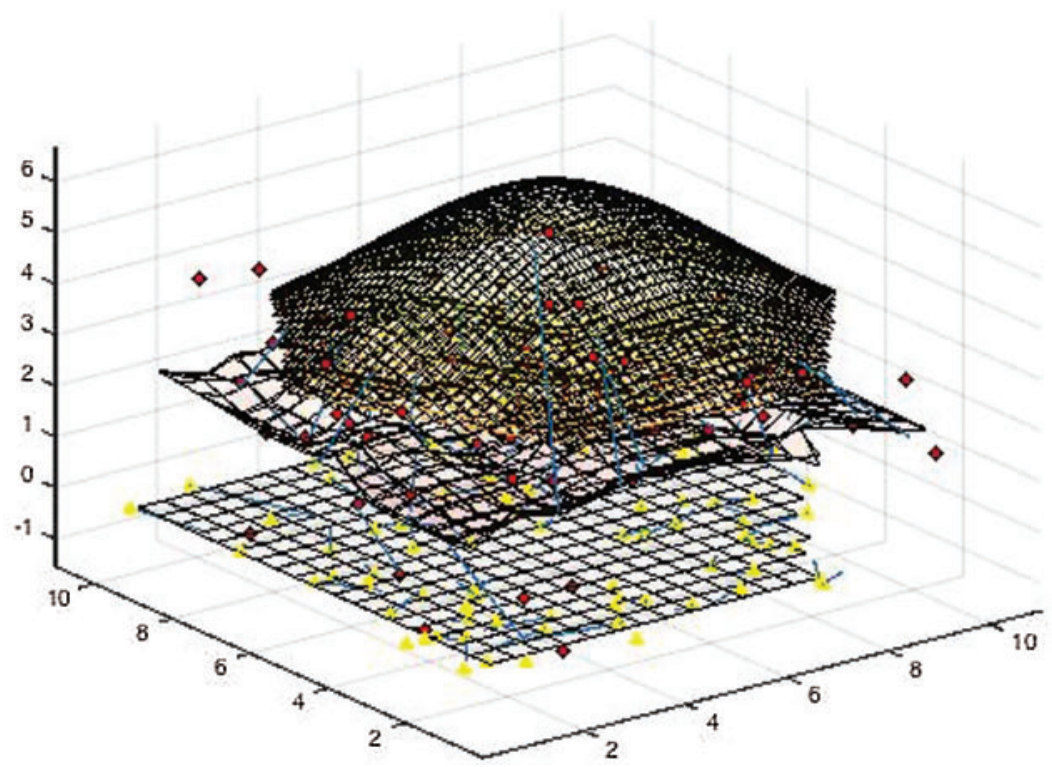

Figure 11: Optimized localization of sensor nodes with HPSO

The localization optimization using algorithms viz. PSO, HPSO, BBO, GWO and FA are already available in the literature with static scenarios. In this paper, these algorithms are also implemented with the proposed technique having single anchor node with umbrella based projection. Further these algorithms are compared with APPA algorithm, given by Tab. 2. 


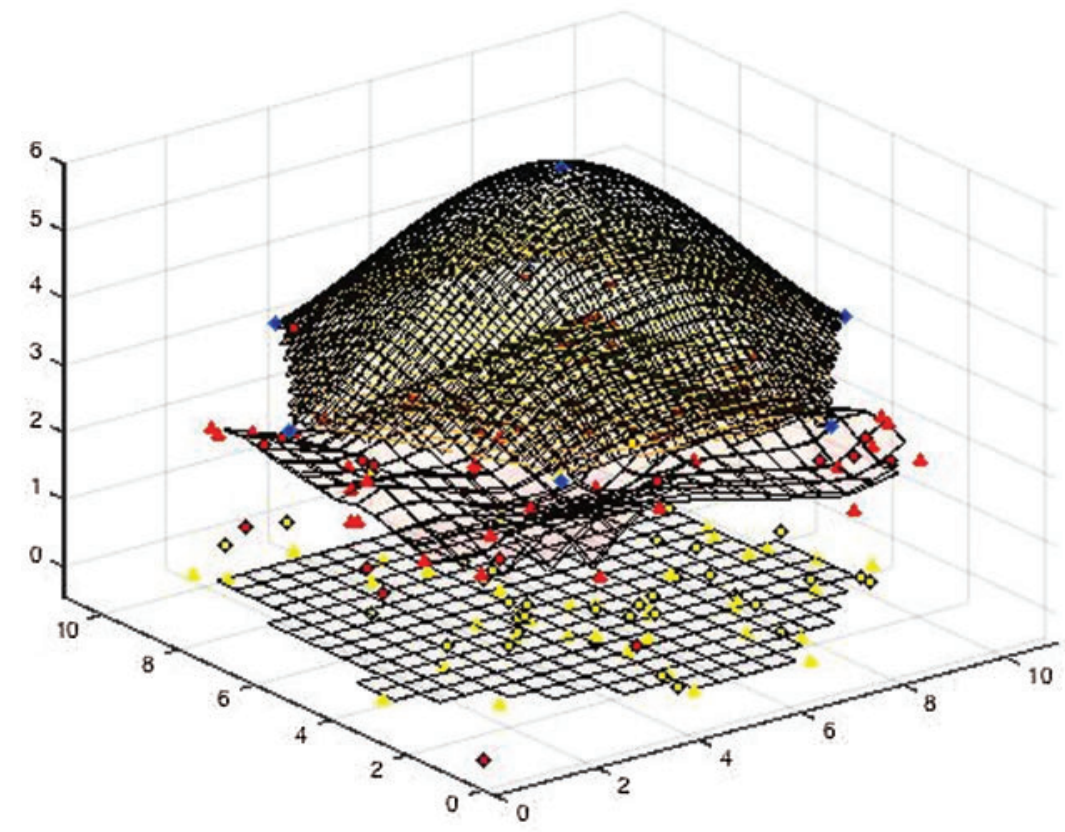

Figure 12: Optimized localization of sensor nodes with GWO

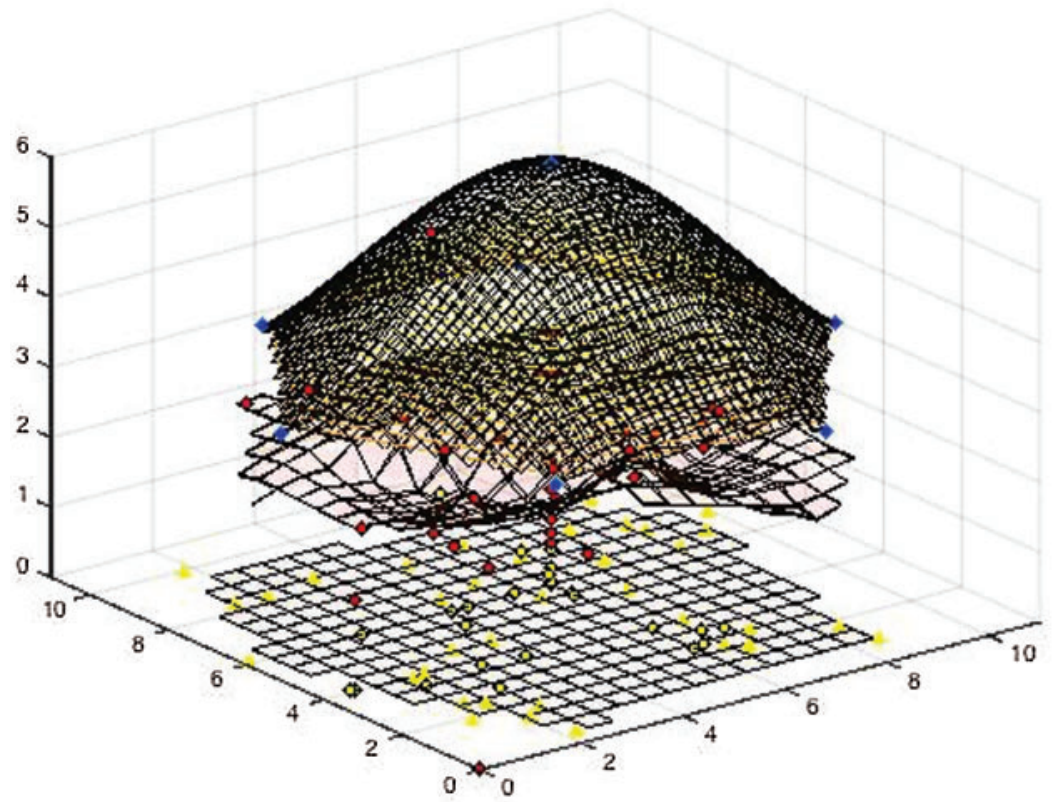

Figure 13: Optimized localization of sensor nodes with APPA

The performances of all algorithms have been compared with the proposed scheme in dynamic scenarios. It has been analyzed from the results given in Tab. 2 that the Average Localization error is coming out to be the minimum for all the various number of movements when we are using APPA Algorithm. 
Table 2: Comparison of meta-heuristic algorithms

\begin{tabular}{|c|c|c|c|c|c|}
\hline Algorithms & $\begin{array}{l}\text { Movements } \\
\text { number }\end{array}$ & $\begin{array}{l}\text { Max localization } \\
\text { error }\end{array}$ & $\begin{array}{l}\text { Min localization } \\
\text { error }\end{array}$ & $\begin{array}{l}\text { Average } \\
\text { error }\end{array}$ & $\begin{array}{l}\text { Number of } \\
\text { located targets }\end{array}$ \\
\hline \multirow[t]{5}{*}{ PSO } & 1 & 3.9358 & 0.0554 & 0.9958 & 80 \\
\hline & 2 & 5.3379 & 0.0831 & 0.9839 & 80 \\
\hline & 3 & 5.0108 & 0.0800 & 0.9267 & 80 \\
\hline & 4 & 5.1655 & 0.0367 & 0.9757 & 80 \\
\hline & 5 & 5.1325 & 0.0812 & 0.9612 & 80 \\
\hline \multirow[t]{5}{*}{ HPSO } & 1 & 3.1204 & 0.1044 & 0.6742 & 80 \\
\hline & 2 & 5.0134 & 0.0647 & 0.4876 & 80 \\
\hline & 3 & 4.8279 & 0.0976 & 0.4032 & 80 \\
\hline & 4 & 5.2376 & 0.0230 & 0.5546 & 80 \\
\hline & 5 & 5.2134 & 0.0316 & 0.5324 & 80 \\
\hline \multirow[t]{5}{*}{$\mathrm{BBO}$} & 1 & 5.8904 & 0.1822 & 1.1892 & 80 \\
\hline & 2 & 5.3500 & 0.3318 & 1.2560 & 80 \\
\hline & 3 & 5.5989 & 0.1822 & 1.1585 & 80 \\
\hline & 4 & 5.6348 & 0.1528 & 1.2818 & 80 \\
\hline & 5 & 5.9014 & 0.1911 & 1.1916 & 80 \\
\hline \multirow[t]{5}{*}{ GWO } & 1 & 3.1101 & 0.0944 & 0.6442 & 80 \\
\hline & 2 & 4.9834 & 0.0547 & 0.4776 & 80 \\
\hline & 3 & 4.8134 & 0.0876 & 0.3932 & 80 \\
\hline & 4 & 4.7976 & 0.0430 & 0.4946 & 80 \\
\hline & 5 & 4.9776 & 0.0513 & 0.4713 & 80 \\
\hline \multirow[t]{5}{*}{ FA } & 1 & 6.1101 & 0.1922 & 2.2234 & 80 \\
\hline & 2 & 6.3120 & 0.3412 & 2.3124 & 80 \\
\hline & 3 & 6.6990 & 0.1923 & 2.4651 & 80 \\
\hline & 4 & 6.8912 & 0.1627 & 2.5123 & 80 \\
\hline & 5 & 6.9036 & 0.2010 & 2.2013 & 80 \\
\hline \multirow[t]{5}{*}{ APPA } & 1 & 3.1101 & 0.0964 & 0.6415 & 80 \\
\hline & 2 & 4.3983 & 0.0437 & 0.4732 & 80 \\
\hline & 3 & 4.8032 & 0.0721 & 0.3841 & 80 \\
\hline & 4 & 4.7679 & 0.0412 & 0.4471 & 80 \\
\hline & 5 & 4.3108 & 0.0403 & 0.4312 & 80 \\
\hline
\end{tabular}

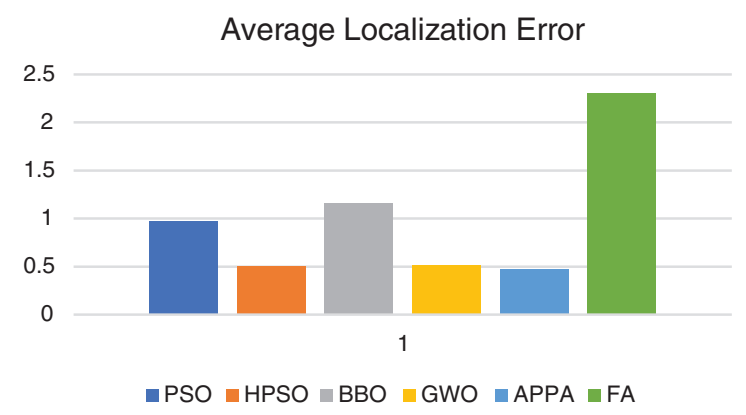

Figure 14: Comparison of average localization error for all the six algorithms 


\section{Conclusions}

The single anchor node method was used to obtain three Dimensional positions of unknown nodes with range-based technique using a meta-heuristic algorithm called APPA. The idea of an anchor and virtual anchor node forms an umbrella projection for finding all unknown nodes. When the mobile target nodes come under the range of the known node, further, with the help of anchor as well as virtual anchors, position of unknown nodes is determined (to find out three dimensional positions, at least four anchor nodes are required). A variety of applications exists where sensor node location is essential and the proposed algorithm is helpful, including logistics, underwater scenarios, tracking of coal mine workers, monitoring of environmental aspects, localization of occurring events in remote and hilly regions etc. Performance of APPA algorithm proposed in this work in order to find out the exact location of the nodes is found out to be better than its competitive algorithms. It has been proved with the help of the results that using APPA, accurate locations are being found as compared to other algorithms and convergence characteristics are also faster. In future, with the help of hybridization of few optimized algorithms, more accuracy could be achieved.

Funding Statement: This research was supported by X-mind Corps program of National Research Foundation of Korea (NRF) funded by the Ministry of Science, ICT (No. 2019H1D8A1105622) and the Soonchunhyang University Research Fund.

Conflicts of Interest: The authors declare that they have no conflicts of interest to report regarding the present study.

\section{References}

[1] R. Kulkarni, G. Venayagamoorthy and M. Cheng, "Bio-inspired node localization in wireless sensor networks," in 2009 IEEE Int. Conf. on Systems, Man and Cybernetics, vol. 22, pp. 205-210, 2009.

[2] D. Lavanya and S. K. Udgata, "Swarm intelligence based localization in wireless sensor networks," in Int. Workshop on Multi-Disciplinary Trends in Artificial Intelligence, Berlin, Heidelberg, Springer, pp. 317-328, 2011.

[3] P. Singh, B. Tripathi and N. Singh, "Node localization in wireless sensor networks," International Journal of Computer Science and Information Technologies, vol. 2, no. 6, pp. 2568-2572, 2011.

[4] A. Boukerche, H. Oliveira, E. Nakamura and A. Loureiro, "Localization systems for wireless sensor networks," IEEE Wireless Communications, vol. 14, no. 6, pp. 6-12, 2006.

[5] Y. Liu, Z. Yang, T. Ning and W. Hongyi, "Efficient quality-of-service (QoS) support in mobile opportunistic networks," IEEE Transactions on Vehicular Technology, vol. 63, no. 9, pp. 4574-4584, 2014.

[6] Y. Liu, Z. Yang, T. Ning and W. Hongyi, "Efficient data query in intermittently-connected mobile ad hoc social networks," IEEE Transactions on Parallel and Distributed Systems, vol. 26, no. 5, pp. 13011312, 2014.

[7] S. Chu, Z. Du and J. Pan, "Symbiotic organism search algorithm with multi-group quantum-behavior communication scheme applied in wireless sensor networks," MDPI, vol. 10, no. 3, pp. 930-952, 2020.

[8] D. Liu, S. Guo, W. Chen and F. Wang, "History based multi-node collaborative localisation in mobile wireless ad hoc networks," International Journal of Ad Hoc and Ubiquitous Computing, vol. 30, no. 2, pp. 59-72, 2019.

[9] S. Kotwal, S. Gill and K. Saini, "Development of range free three dimensional localisation in wireless sensor networks," International Journal of Sensor Networks, vol. 31, no. 1, pp. 52-63, 2019.

[10] K. Low, H. Nguyen and H. Guo, "Optimization of sensor node locations in a wireless sensor network," in Fourth Int. Conf. on Natural Computation, IEEE, vol. 5, pp. 286-290, 2008. 
[11] Y. Wang, P. Wang, J. Zhang, X. Cai, W. Li et al., "A novel DV-hop method based on coupling algorithm used for wireless sensor network localisation," International Journal of Wireless and Mobile Computing, vol. 16, no. 2, pp. 128-137, 2019.

[12] J. Graefenstein, A. Albert, P. Biber and A. Schilling, "Wireless node localization based on RSSI using a rotating antenna on a mobile robot," in 6th Workshop on Positioning, Navigation and Communication, Hannover, Germany, IEEE, pp. 253-259, 2009.

[13] R. Sumathi and R. Srinivasan, "RSS-Based location estimation in mobility assisted wireless sensor networks," in Proc. of the 6th IEEE Int. Conf. on Intelligent Data Acquisition and Advanced Computing Systems, vol. 2, no. 3, pp. 848-852, 2011.

[14] Z. Guo, Y. Guo, F. Hong, Z. Jin, Y. He et al., "Perpendicular intersection: Locating wireless sensors with mobile beacon," IEEE Transactions on Vehicular Technology, vol. 59, no. 7, pp. 3501-3509, 2010.

[15] Q. Shi, H. Huo, T. Fang and D. Li, "A 3d node localization scheme for wireless sensor networks," IEICE Electron Exp., vol. 6, no. 3, pp. 67-72, 2009.

[16] L. Wang, J. Zhang and D. Cao, "A new 3-dimensional dv-hop localization algorithm," Journal of Computing Information Systems, vol. 8, no. 6, pp. 2463-75, 2012.

[17] Y. Xu, Y. Zhuang and J. Gu, "An improved 3D localization algorithm for the wireless sensor network," International Journal of Distributed Sensor Networks, vol. 11, no. 6, pp. 1-13, 2015.

[18] J. Li, X. Zhong and I. Lu, "Three-dimensional node localization algorithm for WSN based on differential RSS irregular transmission model," Journal of Communications, vol. 9, no. 5, pp. 391-397, 2014.

[19] T. Ahmad, X. Li and B. Seet, "Parametric loop division for 3D localization in wireless sensor networks," Sensors, MDPI, vol. 17, no. 7, pp. 1697-1729, 2017.

[20] A. Gopakumar and L. Jacob, "Localization in wireless sensor networks using particle swarm optimization," in IET Conf. on Wireless, Mobile and Multimedia Networks, Beijing, China, pp. 227-230, 2008.

[21] P. Chuang and C. Wu, "An effective pso-based node localization scheme for wireless sensor networks," in Ninth Int. Conf.on Parallel and Distributed Computing, Applications and Technologies, Dunedin, New Zealand, IEEE, pp. 187-194, 2008.

[22] J. Kennedy, "Bare bones particle swarms," in Swarm Intelligence Sym., Indianapolis, IN, USA, IEEE, pp. 80-87, 2003.

[23] R. Kulkarni and G. Venayagamoorthy, "Particle swarm optimization in wireless-sensor networks a brief survey," IEEE Transactions on Systems, Man, and Cybernetics, Part C (Applications and Reviews), vol. 41, no. 2, pp. 262-267, 2011.

[24] A. Kumar, A. Khosla, J. Saini and S. Singh, "Meta-heuristic range-based node localization algorithm for wireless sensor networks," in Int. Conf. on Localization and GNSS, Starnberg, Germany, IEEE, pp. $1-7,2012$.

[25] S. Arora and S. Singh, "Node localization in wireless sensor networks using butterfly optimization algorithm," Arabian Journal of Science and Engineering, vol. 42, no. 8, pp. 1-11, 2017.

[26] P. Singh, A. Khosla, A. Kumar and M. Khosla, "A novel approach for localization of moving target nodes in wireless sensor networks," International Journal of Grid and Distributed Computing, vol. 10, no. 10, pp. 33-44, 2017.

[27] P. Singh, A. Khosla, A. Kumar and M. Khosla, "Computational intelligence based localization of moving target nodes using single anchor node in wireless sensor networks," Telecommunication Systems, Springer vol. 69, no. 3, pp. 397-411, 2018.

[28] P. Singh, A. Khosla, A. Kumar and M. Khosla, "3D localization of moving target nodes using single anchor node in anisotropic wireless sensor networks," AEU-International Journal of Electronics and Communications, vol. 82, no. 1, pp. 543-552, 2017. 
[29] P. Singh, A. Khosla, A. Kumar and M. Khosla, "Optimized localization of target nodes using single mobile anchor node in wireless sensor network," AEU-International Journal of Electronics and Communications, vol. 91, no. 3, pp. 55-65, 2018.

[30] P. Singh, A. Khosla, A. Kumar and M. Khosla, "A novel approach for localization of moving target nodes in wireless sensor networks," International Journal of Grid and Distributed Computing, vol. 10, no. 10, pp. 33-44, 2017. 